\title{
On the issue of reducing the negative impact of nitrogen oxides in the system recirculation of diesel exhaust gases on applying the vortex effect
}

\author{
Irina Belinskaia ${ }^{1 *}$, Rahim Zainetdinov ${ }^{2}$, and Konstantin Evdokimov ${ }^{3}$ \\ ${ }^{1}$ The Leningrad State University after named A.S. Pushkin Peterburgskoe shosse, 10, Saint- \\ Petersburg-Pushkin, Russia \\ ${ }^{2}$ Saint-Petersburg State Agrarian University Peterburgskoe shosse, 2, Saint-Petersburg-Pushkin, \\ Russia \\ ${ }^{3}$ Belgorod University of Cooperation, Economics \& Law, 308023, Belgorod, Sadovaya st, 116a, \\ Russian Federation
}

\begin{abstract}
The problem of negative impact on the environment of motor transport is one of the most fundamental in the complex of global problems. The constant increase in the number of cars with internal combustion engines encourages the search for methods and ways to reduce the volume of negative impulses. The operation of heat engines is accompanied by significant emissions of gaseous harmful substances into the atmosphere, i.e. nitrogen oxides, carbon monoxide, hydrocarbons, as well as solid particles, including soot. The solution to this problem should be implemented within the framework of a systematic approach. To do this, it is necessary to combine the study of technical, economic, and organizational approaches to the organization of the exhaust gas disposal process. To date, there is a significant methodological base in the field of organizational and economic decisions. The article discusses various methods of cleaning exhaust gases of piston engines, their advantages and disadvantages are noted. The method of processing using ammonia is widely known. It is noted that a catalytic method for reducing nitrogen oxides using ammonia is quite economical. However, the optimal temperature range at which nitrogen oxides are reduced is rather narrow. To solve this problem, it is proposed to use the vortex effect in the exhaust system. The efficiency of using a vortex gas recirculation pipe is due to its significant influence on the thermal gasdynamic processes occurring in the exhaust system. Using the principles of non-equilibrium thermodynamics allows us to take into account dissipative processes when establishing the relationship of fuel and economic indicators of internal combustion engines with thermodynamic parameters. This significantly increases the accuracy of calculations and allows you to develop measures to reduce the level of negative impact on the environment.
\end{abstract}

* Corresponding author: belinska@yandex.ru 


\section{Introduction}

Environmental issues are becoming more and more acute. At the same time, one of the sources of toxic substances released into the atmosphere is diesel power plants (DPP). Harmful emissions from reciprocating power plants cause irreparable harm to human health, construction buildings and structures built by them, as well as to the environment, reduce yields (up to $25 \%$ ) and degrade the quality of crops, especially near major highways $[1,2$, 3].

Exhaust gases (EG) of DPP are a complex multicomponent mixture of gases that contain more than 250 components, including products of complete fuel combustion and substances formed at high temperatures (nitrogen oxides ((NOx), products of incomplete fuel combustion (carbon monoxide, hydrocarbons , soot)), as well as sulfur oxides, aldehydes, etc. $[1,2]$. Therefore, the issue of improving the environmental performance of piston engines, along with economic ones, is a major task.

Analysis of the main factors affecting the formation of harmful substances in diesel engines has shown that due to the peculiarities of the working process occurring in them, a decrease in emissions of incomplete combustion products, carbon oxides, hydrocarbons and harmful particles is accompanied by an increase in emissions of nitrogen oxides and by contrast $[1,2,4,5,6]$. Therefore, to ensure that diesel meets current and future standards for harmful emissions, exhaust gas recirculation and a catalytic method for their recovery are widely used to reduce nitrogen oxide emissions $[7,8,9,10,11,12]$.

The aim of the study is to reduce the nitrogen oxides of piston engines by using a vortex tube in the exhaust gas recirculation system, taking into account the non-equilibrium of gas dynamic processes.

It should be noted that the ways to improve the environmental safety of diesel engines by using exhaust gas recirculation and their neutralization show that the effectiveness of their use depends on the temperature of the recirculated gases (RG) and the temperature of the exhaust gases $[8,9,12,13,14,15]$, characterized by a significant mass, metal content (nonferrous metals) and having significant hydraulic resistance.

It is known that an important feature of the catalytic method for reducing nitrogen oxides using ammonia is the presence of a narrow optimal temperature range (1000-1500K), in which the reduction of $N O$ is effectively ensured with the formation of molecular nitrogen $N_{2}[16,17]$.

\section{Methods and methodology}

Meanwhile, the materials of the works $[9,18]$ they indicate the theoretical possibility of using a vortex tube to partially solve the problem noted above. At the same time, most of the time diesel power plants operate at variable speed and load modes, and therefore the temperature of the exhaust gases (and hence the recirculated gases) varies widely (from 120 to $7500 \mathrm{C}$ ). Therefore, to ensure the most profitable process of reducing nitrogen oxides $N O_{X}$ with ammonia in the neutralizer, it is necessary to raise the exhaust gas temperature to the optimal temperature range, taking into account heat exchange in the exhaust manifold and engine operating modes. As devices that increase the temperature waste gases before entering the catalytic neutralizer, it is possible to use a vortex tube where the temperature rise of exhaust gases in front of the catalyst based on the use of vortex effect with the separation of exhaust gases into two streams - heated and cooled.

Compressed exhaust gases at pressure and temperature $T_{B X}$ enter the pipe, and perform a rotational movement relative to the pipe axis and moves along the periphery of the pipe from the nozzle section to the hot end, and complex gas-dynamic and thermal processes occur, depending on the shape and ratio of the geometric dimensions of the pipe elements 
(Fig. 1). In this case, an uneven temperature field occurs and the gas layers located near the axis are colder than the incoming compressed exhaust gas, and the peripheral layers of the swirling flow are heated and directed towards the hot end of the pipe [19, 20]. The proportion of cold recirculated gases sent to the intake manifold is regulated by the vortex tube throttle valve, which provides the necessary cooling of the recirculated gases depending on the operating mode of the power plant.

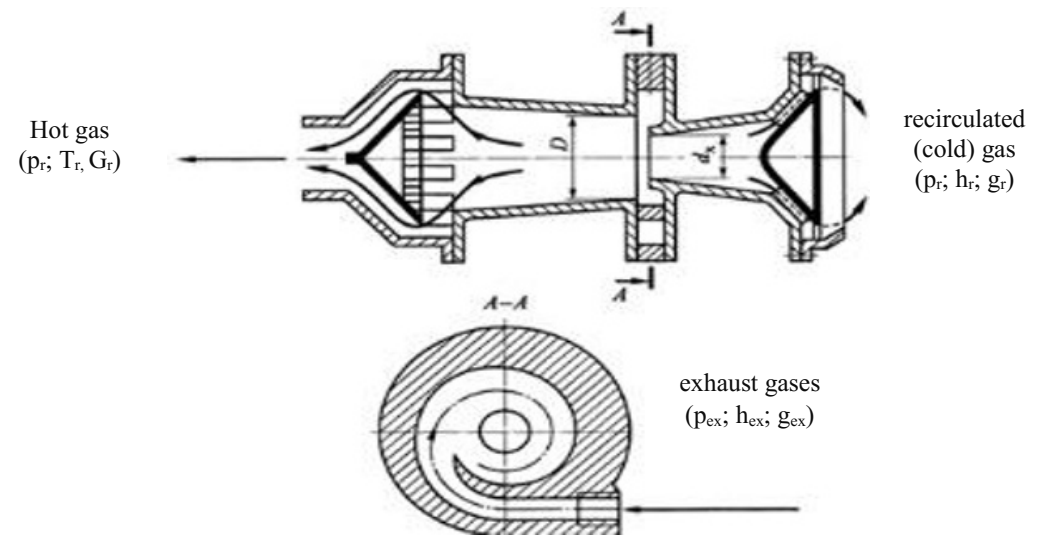

Fig. 1. Functional diagram of a vortex tube.

\section{Results and discussion}

A thermodynamic study of a vortex tube is usually performed at known values of the thermodynamic parameters in front of the vortex tube, which depend on the thermogasodynamic processes occurring in the exhaust system of a diesel power plant.

In calculations, the gas flow is assumed to be stationary if there are losses in the heat exchange process between the pipeline walls and the gas. The gas velocity at its entrance to the inlet pipeline is considered subsonic. The gas is assumed to be perfect, so that at each point its pressure $\mathrm{p}$, density $\mathrm{p}$, and temperature $\mathrm{T}$ are related by the equation of state:

$$
p=\rho R T .
$$

Flow continuity equations along the entire outlet channel have the form:

$$
G_{\mathrm{or}}=\rho w F=\text { const, }
$$

where $F$ - the cross-sectional area of the outlet; $w$ - gas flow velocity.

During heat exchange of the gas flow in the exhaust pipeline with the external environment heat removal $Q_{s h}$ it leads to a change in the gas parameters:

$$
d Q_{s h}=d h+d\left(\frac{w^{2}}{2}\right)
$$

where $d Q_{s h}$-the amount of heat removed from the exhaust system pipeline; $d h$ and $d\left(w^{2} / 2\right)$ - accordingly, the change in the enthalpy and kinetic energy of the exhaust gases.

Heat removal through the elementary length $d x$ of the pipeline is equal

$$
\frac{d Q_{s h}}{d x}=c_{p} \frac{d}{d x}\left(T+\frac{w^{2}}{2 c_{p}}\right)
$$

Here can enter the following symbols:

$$
q_{\mathrm{\Gamma}}=\frac{G_{0 \Gamma}}{S_{h p}} \frac{d Q_{c m}}{d x}, \quad T^{*}=T+\frac{w^{2}}{2 c_{p}} .
$$

where $q_{\Gamma}$ - heat flux density; $S_{\text {об }}$ - heated perimeter of the pipe; $T^{*}$ - braking temperature; $c_{p}$ - Isobaric heat capacity of exhaust gases. 
Substituting the ratio $q_{\mathrm{\Gamma}}$ and $T^{*}$ in equation (4), can get the following expression:

$$
\frac{\mathrm{dT}^{*}}{\mathrm{dx}}=\frac{\mathrm{S}_{\mathrm{hp}}}{\mathrm{G}_{\mathrm{or}} \mathrm{c}_{\mathrm{p}}} \mathrm{q}_{\mathrm{r}} \text {. }
$$

Heat removal from exhaust gases to the environment (4) is accompanied by a decrease in the gas braking temperature.

To close a system of equations (1), (2), (5) and (6), the generalized Bernoulli equation is used [21]:

$$
\frac{1}{\rho} \frac{d p}{d x}+w \frac{d w}{d x}+\xi \frac{w^{2}}{2 D}=0
$$

where $D$ - outlet pipe diameter (hydraulic diameter); $\xi$ - resistance coefficient of the gas flow outlet line under consideration.

A system of five equations (1), (2), (5), (6) and (7) contains five quantities to be determined $T, p, \rho, w, T^{*}$. In this case, the gas consumption $G_{w g}$ and its heat capacity $c_{p}$ are known values. The value of the specific heat flow of exhaust gases $q_{\Gamma}$, cross-section area of the exhaust pipeline $F$, heated perimeter $S_{0}$ are knows also. From equation (6), it is possible to determine the braking temperature $\mathrm{T}^{*}$ in any section of the pipeline:

$$
T^{*}=\frac{1}{G_{\text {ug }} c_{p}} \int_{0}^{x} \overrightarrow{q_{\mathrm{\Gamma}}} S_{0} d x+T_{0}^{*} .
$$

Maha number $(M=w / a)$ can be represented as:

$$
M^{2}=\frac{w^{2}}{k R T}
$$

where $k=c_{p} / c_{v}$-adiabatic index; $w$ - gas flow velocity.

Expression (5) can be converted to the form:

$$
\frac{T^{*}}{T}=1+\frac{k-1}{2} M^{2}
$$

Given equality (1), the Bernoulli equation (7) can be written as:

$$
\frac{1}{p} \frac{d p}{d x}+\frac{k M^{2}}{w} \frac{d w}{d x}+\xi \frac{k M^{2}}{2 D}=0
$$

Equation (1) after differentiating both its parts has the form:

$$
\frac{1}{p} \frac{d p}{d x}=\frac{1}{T} \frac{d T}{d x}+\frac{1}{\rho} \frac{d \rho}{d x}
$$

Similarly, equation (2) can be expressed in differential form as:

$$
\frac{1}{\rho} \frac{d \rho}{d x}=\frac{1}{w} \frac{d w}{d x}-\frac{1}{F} \frac{d F}{d x}
$$

Equality (9) after differentiation will take the form:

$$
\frac{1}{\rho} \frac{d \rho}{d x}=\frac{1}{w} \frac{d w}{d x}-\frac{1}{F} \frac{d F}{d x}
$$

Substituting the obtained differential equations (13) and (14) in (12), obtain the following expression:

$$
\frac{1}{p} \frac{d p}{d x}=\frac{1}{w} \frac{d w}{d x}-\frac{1}{M^{2}} \frac{d M^{2}}{d x}-\frac{1}{F} \frac{d F}{d x}
$$

The Bernoulli equation (11), after inserting the expression (15), will take the form:

$$
\frac{1+k M^{2}}{w} \frac{d w}{d x}-\frac{1}{M^{2}} \frac{d M^{2}}{d x}+\frac{\xi k M^{2}}{2 D}-\frac{1}{F} \frac{d F}{d x}=0
$$

Using expressions (9) and (10), we can find:

$$
w^{2}=k R T^{*} \frac{M^{2}}{1+\frac{k-1}{2} M^{2}} .
$$

From where, after differentiating expression (17), we can get an equation of the following form:

Substituting the right side of this equation in expression (16) and after integration we get the expression:

$$
\frac{1}{w} \frac{d w}{d x}=\frac{1}{2 T^{*}} \frac{d T^{*}}{d x}+\frac{1}{2 M^{2}\left(1+\frac{k-1}{2} M^{2}\right)} \frac{d M^{2}}{d x} .
$$


Substituting the right side of this equation in expression (16) and after integration we get the expression

$$
\begin{aligned}
& \frac{1}{2} \ln M^{2}-\frac{(k+1)}{2(k-1)} \ln \left((k-1) M^{2}+2\right)-\frac{1+k M^{2}}{2} \ln T^{*}- \\
& -\xi \frac{k M^{2}}{2 D} x+\ln F+C=0
\end{aligned}
$$

Next, the temperature $T$ can be found using equation (10), the speed of the gas flow $w-$ the expression (9), and the pressure $p$ and density $\rho$ determined from equations (1) and (2).

It is known that the exhaust gases at the exit of the cylinder have a sufficiently high efficiency, which is realized in the form of work or heat. When using a vortex tube, some of this energy is used for the exhaust gas separation process.

When evaluating the energy efficiency of exhaust gases, the exergetic method is usually used, but all thermogasodynamic processes in the exhaust system are irreversible, and the amount of energy dissipated is determined by the production of entropy. Therefore when evaluating the efficiency of exhaust gas energy use, the exergetic method using the principles of non-equilibrium thermodynamics is more promising. Then the exergy differential for such a system can be represented as

$$
d E=d H-T_{0} d S,
$$

where $H$ - enthalpy of a thermodynamic system; $T_{0}$ - ambient temperature.

Due to the fact that the exhaust gases are inert, so they can be considered as a continuous homogeneous open thermodynamic system, then the expression of the enthalpy differential can be written as:

$$
d H=T d S+V d p \text {. }
$$

where $T, V, p$ - according: temperature, volume and pressure of the exhaust gases.

Substituting it into equation (20), we can get:

$$
d E=\left(T_{\text {ог }}-T_{0}\right) d S+V d p \text {. }
$$

From expression (22), it is possible to determine the rate of change in the specific exergy of exhaust gases:

$$
\frac{d e}{d t}=\left(T_{\text {ог }}-T_{0}\right) \frac{d s}{d t}+v \frac{d p}{d t}
$$

The ratio of entropy production in the absence of a chemical reaction in the system can be written as [22]:

$$
\rho \frac{d s}{d t}=-d i \vec{v}_{s}+\sigma_{s}^{\text {or }}
$$

where $\vec{J}_{S}-$ the total entropy flow vector in the system under consideration; $\sigma_{\Gamma}-$ local rate of entropy occurrence due to internal irreversible processes in the exhaust pipeline.

From expression (24), it follows that changes in the entropy of the gas flow between the outlet sections of the engine valve mechanism and the inlet of the engine vortex tube due to the transfer of entropy with the heat of the exhaust gases and due to the production of entropy in internal non-equilibrium processes. The total entropy flow vector $\overrightarrow{J_{s}}$ consists of two parts: convective and entropy flow due to heat conduction processes

$$
\overrightarrow{J_{s}}=\rho s \vec{W}-\frac{\vec{q}_{u s}}{T},
$$

where $\vec{W}$-is the total velocity vector of the gas flow; $q_{w s} \quad$ - is the heat flux density on the pipeline wall from the side of the hot exhaust gas flow.

When idealizing processes in gas flows, it is quite acceptable to neglect friction, since the viscosity of gases is small, and the length of channels in technical devices for energy conversion is relatively small. It is unacceptable to ignore the influence of non-equilibrium gas expansion, since gas expansion is inseparable from the very nature of the gas flow. The process of expansion of the gas flow is a polytropic process, therefore, to calculate the change in entropy in the gas flow, you can use the equation [23]:

$$
d_{e} S=c_{v} \frac{M^{2}-1}{M^{2}} \frac{d T}{T}
$$


where $M-$ Mach number ; $c_{v}$ - heat capacity at constant volume.

Usually, when the gas flow flows in the outlet pipeline, the flow temperature value gradually decreases. Analyzing the dependence (11), it is easy to see that for subsonic flow $(\mathrm{M}<1)$, the entropy increases, and for supersonic flow $(\mathrm{M}>1)$ it decreases. The flow of gas at supersonic speed in the channels of a constant cross-section of the exhaust pipeline is unattainable in the absence of external influences.

Gas expansion in the internal combustion engine valve device occurs isothermically with an increase in entropy:

$$
d_{e} S_{\text {кл }}=R \ln \left(v_{2} / v_{1}\right)
$$

Heat flow $\underset{q_{g}}{\rightarrow}$ on the wall of the pipeline from the side of the exhaust gases hot flow can be represented as [24]:

$$
\vec{q}_{\Gamma}=S t \cdot c_{p} \cdot \rho \cdot \vec{W} \cdot\left(T_{\mathrm{cp}}-T_{W}\right)
$$

where $T_{\mathrm{cp}}$ - average cross-section thermodynamic temperature of the gas stream; $T_{W}-$ wall temperature on the hot gas side; $S t$ - the number of Stanton.

When calculating the heat exchange with the outlet pipe wall, the Stanton number can be determined from the expression [21]:

$$
S t_{1}=\left[\frac{8}{\lambda_{1}}+11,5(\operatorname{Pr}-1) \sqrt{\frac{8}{\lambda_{1}}}\right]
$$

where $\lambda_{1}-$ drag coefficient ; Pr - criterion of Prantdl.

In the exhaust system, additional sources of entropy generation due to internal dissipative processes are the processes of thermal conductivity, heat energy dissipation due to friction and gas throttling. Entropy also increases as a result of the pressure drop during the exhaust gas flow through the exhaust pipe of the exhaust system. The local rate of occurrence of entropy $\sigma_{S}$ due to internal irreversible processes in the system under consideration has the form:

$$
\sigma_{S i}=\frac{1}{T^{2}} \vec{q} \nabla T-\frac{1}{T_{m i}} \vec{J}_{\text {ог }}\left(T_{m i} \cdot \operatorname{grad} \frac{\mu_{k}}{T_{m i}}-\vec{F}_{k}\right),
$$

where $J_{\text {ог }}$ - the vector flow of exhaust gases, $F_{k}$ - component responsible for barodiffusion (transfer of the gas flow due to the pressure gradient), $\vec{F}_{k}=\vec{v}_{k} \nabla p ; \quad T_{m i}=$ $\left(T_{i}^{\prime \prime}-T_{i}^{\prime}\right) / \ln T_{i}^{\prime \prime} / T_{i}^{\prime}$ - average temperature; $T^{\prime}$ и $T^{\prime \prime}$ - accordingly, the temperature at the inlet and outlet of the $i$-th section of the exhaust pipeline.

The expression of the local entropy production $d_{i} S / d t$ when passing through the pipeline with heat exchange, it will be reduced to the form:

$$
\sigma_{w g}=\frac{d_{i} S}{d t}=q_{g}\left(T_{g}, T_{c_{1}}\right)\left(\frac{1}{T_{m i}}-\frac{1}{T_{W}}\right)
$$

where $q_{g}$ - heat flux supplied to the surface of the collector from the exhaust gases.

When gas passes through the valve device of the gas distribution mechanism, the gas pressure changes, and its flow rate depends on the cross-section time and pressure drop. Assuming the flow process is isothermal, the expression entropy production when gases are throttled through the valve slot can be written as:

$$
\sigma_{t h}=\frac{1}{t} \int_{0}^{t} G_{g}\left(p_{b}, p_{2}\right) \frac{\mu_{1}\left(p_{1}, v_{1}, T\right)-\mu_{2}\left(p_{2}, v_{2}, T\right)}{T} d t
$$

where $\mathrm{G}_{\mathrm{g}}\left(p_{b}, p_{2}\right)$ - gas flow through the valve device of the gas exchange system; $p_{b}-$ pressure in the cylinder when the exhaust valve starts opening; $p_{2}$ - gas pressure in the exhaust manifold behind the valve

Mass flow rate of combustion products when passing through the valve device

$$
G_{g}=\mu f_{k} \cdot \sqrt{\frac{2 k}{k-1} p_{b} v_{b}\left[1-\left(\frac{p_{2}}{p_{b}}\right)^{\frac{k-1}{k}}\right]},
$$

where $\mu$ - flow coefficient; $f_{k}-$ the average cross-sectional area of the exhaust valve. 
It follows from equation (30) that the increase in entropy due to the pressure drop during the flow of exhaust gases through the exhaust pipeline is equal to

The pressure drop in the pipeline $\nabla p_{i}$ is proportional to the square of the average flow rate

$$
\nabla p_{i}=\lambda \rho \cdot \frac{W^{2} d x}{2 D}
$$

where $x$-coordinate indicating the distance from the beginning of the pipeline and to any of its sections; $\lambda$-the coefficient of resistance, the numerical value of which depends on the nature of the gas flow and on the Reynolds number.

The estimated pressure retractably gases $p_{w g}$, temperature $T_{w g}$ supplied to the vortex tube exhaust and the given values of pressure $p_{\Gamma}$ hot, cold $p_{X}$ flows and the mass fraction of cold gas flow calculation $\mu$ process ideal vortex tube is as follows [19].

The temperature of the cold stream is determined by the formula:

$$
T_{X}=T_{C} \cdot \tau_{C . X .}=T_{C} \cdot\left(\frac{p_{X}}{p_{C}}\right)^{(k-1) / k} .
$$

The temperature of the hot stream is calculated from the expression

$$
T_{g}=T_{C} \frac{1-\mu \cdot \tau_{C . X} .}{1-\mu} .
$$

Increase in specific entropy $\Delta s_{h s}$ in the hot flow in the vortex tube is equal to

$$
\Delta s_{h s}=R \ln \left(p_{b} / p_{g}\right) \text {, }
$$

where $R$ - gas constant flow.

The pressure $p_{b}$ can be determined from the expression:

$$
p_{b}=p_{C} \frac{1}{\left(\frac{T_{C}}{T_{g}}\right)^{k /(k-1)}}
$$

where $k$ - adiabatic coefficient.

The vortex tube coefficient can be determined from the expression [19]:

$$
\eta_{\text {Ид. }}=1-\frac{(1-\mu) \Delta s_{\Gamma \Pi} T_{\text {ОГ }}}{e_{\text {ВХ }}}
$$

where $e_{v t}-$ specific exergy of exhaust gases at the entrance to the vortex tube.

It should be noted that the solution to the issue of reducing the toxicity of exhaust gases of diesel engines using gas recirculation waste gases is in contradiction with the requirements of better fuel economy due to the deterioration processes of mixture formation and combustion, thus reduction of technical and economic parameters of the internal combustion engine.

When recirculating exhaust gases, the proportion of residual gases in the engine cylinder artificially increases, which increases the duration of the ignition delay and reduces the rate of heat release. Also, when the air charge is heated by recirculated gas flows, especially in non-supercharged engines, the filling coefficient $\eta_{V}$ decreases. The process of mixing fresh air charge flows with recirculating gases having different thermodynamic States and chemical composition occurs due to mutual thermo-and barodiffusion of the components, which is irreversible. The presence of internally irreversible processes in thermodynamic systems causes the appearance of entropy.

In the process of filling the cylinder with a working charge, the main sources of entropy generation due to internal dissipative processes are processes aimed at equalizing the intensive parameters - temperature, pressure, and chemical potentials of the components of the working charge by volume. Non-equilibrium processes associated with heat exchange of the mixed fresh charge with the walls of the intake pipeline, hydraulic resistances of the engine intake path taking into account the exhaust gas recirculation line, including flow separation processes in the vortex tube and throttling of the fresh charge in the valve device, also produce entropies during filling in the intake system.

In this regard, minimizing the reduction in engine power when using the exhaust gas recirculation system involves establishing a connection between the indicator efficiency of 
the engine with the composition of the working charge and irreversible thermogasodynamic processes in the gas exchange system.

It is obvious that minimizing dissipative losses in the processes under consideration provides a minimal reduction in the indicator efficiency $\eta_{i}$, which can be determined using the expression

$$
\eta_{i}=\frac{Q_{1}-Q_{2 \mu}}{Q_{1}}=\frac{\int_{V} p d V}{Q_{1}}
$$

where $Q_{1}$ - heat received from a hot source.

The amount of useful work of the extension can be determined from the expression [23]:

$$
\begin{aligned}
& p d V=\frac{1}{1-\tau_{e}}\left[\tau_{e} d U+\left(1-\tau_{e}\right) \sum_{k} \mu_{k} d N_{k}+p_{0} d V+d E_{\mathrm{BX}}-\right. \\
& \left.-\sum d E_{\mathrm{BЦ}}-d E_{\text {ог }}-\sum_{m} d D_{m}\right],
\end{aligned}
$$

where $p, V, U-$ pressure, volume, and internal energy of a thermodynamic system; $\sum d E_{c}-$ total exergy of heat exchange processes through the cylinder wall, fuel evaporation; $E_{i c}-$ exergy of the working charge at the cylinder inlet; $\tau_{e}-$ temperature exergetic function of available heat; $D_{m}$ - total exergy losses due to the irreversibility of heat generation and heat exchange processes in the over-piston space and thermogasodynamic processes in the exhaust gas pipeline; $d E_{w g}$ - change in the energy of the gas flow in the exhaust path.

Substituting the expression (42) in the formula (41), it is possible to determine the indicator efficiency of the engine, taking into account the non-equilibrium of intra-cylinder processes, and to estimate the degree of heat dissipation in them. It can also be noted that the greater the degree of irreversibility of these processes, the greater the rate of heat dissipation formation in the supra-piston space.

The cylinder power of the engine can be represented as:

$$
\frac{d V}{d t}=\frac{1}{1-\tau_{e}}\left[\tau_{e} \frac{d U}{d t}+\left(1-\tau_{e}\right) \sum_{k} \mu_{k} \frac{d N_{k}}{d t}+p_{0} \frac{d V}{d t}+\frac{d E_{0}}{d t}--\sum \frac{d E_{\mathrm{B}}}{d t}-\frac{d E_{\mathrm{O \Gamma}}}{d t}-\sum_{k} \frac{d D_{n}}{d t}\right]
$$

This formula, taking into account the expression (42), allows us to determine the indicator efficiency of the engine, taking into account the non-equilibrium of intra-cylinder processes, and to assess the degree of heat dissipation in them. It can also be noted that the greater the degree of irreversibility of these processes, the greater the rate of formation of heat dissipation in the supra-piston space.

$\mu_{k} d N_{k}$ - the second term - in the right-hand side of equation (41) is equal to the thermodynamic potential of Gibbs. The integral form of this term has the form

$$
\sum_{k} \int \mu_{k} d N_{k}=\sum_{k} \mu_{k} N_{k}-\frac{H u}{l_{0}+1}
$$

where $l_{0}$ - stoichiometric amount of air; $H u$ - the lowest calorific value, taking into account the concentration of the recirculated gases.

In equation (43), the change in internal energy $U$ can be determined from the relation:

$$
\frac{d U}{d t}=m \frac{d u}{d t}+u \frac{d m}{d t}=m\left(\frac{\partial u}{\partial p}\right)_{T, \alpha_{B}} \frac{d p}{d t}+m\left(\frac{\partial u}{\partial T}\right)_{p, \alpha_{B}} \frac{d T}{d t}
$$

where $m, u$ - mass and specific internal energy of the working body (charge); $Q_{x}(\tau)$ - heat dissipation characteristic; $\alpha_{B}-$ air excess factor.

The change in the excess air coefficient $\alpha_{B}$ during the working process essentially expresses the change in the composition of the working fluid, which is considered as a gas mixture of various components. Gas recirculation is accompanied by a decrease in the maximum cycle temperature due to a decrease in the fresh charge $\left(\alpha_{B}\right)$ and an increase in its heat capacity Naturally, depending on the coefficient of excess air, the internal energy changes, which must be taken into account in the thermodynamic calculation of the working process in the engine of concentration $N O_{x}$. 
Oxides are formed by the combustion of fuel in the engine under high pressures and temperatures, as well as excess oxygen. The description of the kinetics of fuel combustion presents significant challenges. In this regard, in engineering calculations, the well-known dependence is used to determine the concentration of $N O_{x}[2]$ :

$$
\frac{d\left(N O_{x}\right)}{d \tau}=A \frac{\alpha_{\mathrm{B}}-1}{\alpha_{\mathrm{B}}} p \exp \left(-\frac{E}{R T}\right)
$$

where $p$ and $T$ - the pressure and temperature in the cylinder $E$ - activation energy in the formation reaction NOx; $R$ - universal gas constant; $A$ - constant, $A=10^{4}$.

The application of formula (46) is valid when integrating $N O_{x}$ in the kinetic combustion phase. When the conditions in the engine cylinder change, the ratio is taken $E /(R T)=21200$.

\section{Conclusion}

The proposed theoretical development allows us to establish the quantitative dependence of fuel and economic indicators of a diesel power plant on the share of recirculated gases in the fresh charge and on the degree of reduction of their temperature using a vortex tube, speed and load modes of the engine itself. When the engine is running at full load, the exhaust gas recirculation system is switched off.

The use of a vortex tube in the exhaust gas recirculation system allows cooling of recirculated gases, which can replace the use of traditional recuperative heat exchangers under operating conditions. The simultaneous increase in the exhaust gas temperature in front of the neutralizer using a vortex tube provides an additional reduction in nitrogen oxide emission concentrations by using ammonia in the neutralizer. Controlling the exhaust gas temperature using a vortex tube in front of the neutralizer, depending on the speed and load modes of the diesel power plant, allows maintaining an optimal temperature range, in which the reduction of $N O$ with the formation of $N_{2}$ molecules is effectively ensured.

The use of a vortex tube in the exhaust gas recirculation system provides a comprehensive reduction of nitrogen oxides by affecting the combustion process in the engine and creating an optimal condition for further reduction with ammonia in the neutralizer.

For high-speed supercharged diesels with a battery injection system and an electrohydraulic nozzle, the proposed recirculating gas cooling system can be highly efficient while ensuring optimal control of the recirculation degree and creating a vortex effect when separating the exhaust gas flow in the exhaust system.

Application of the vortex tube recirculation gases affect the thermal gas-dynamic processes occurring in the exhaust system. These processes are irreversible, so the assessment of the efficiency of exhaust gases in the proposed scheme by the exergetic method using the principles of non-equilibrium thermodynamics allows us to take into account dissipative processes in establishing the relationship of fuel and economic indicators of internal combustion engines with thermodynamic parameters occurring in the gas exchange system.

\section{References}

1. M.V. A.arkov, R.M. Bashirov, I. I.Gabitov, Toxicity of diesel exhaust gases, M.: Publishing house of MSTU, Bauman, - $376 \mathrm{p}$ (2002)

2. V. I. Erokhova, Toxicity of modern cars, M.: Forum: INFRA-M, 448 p (2013)

3. T. Sencic, Analysis of soot and Nox emission reduction possibilities on modern low speed, two-stoke, diesel engines, Strojarstvo, vol. 52(5), pp. 525-533. 
4. I.A. Berg, S. V.Porshnev, V. Y. Oshchepkova, Pulsation-based method for reduction of nitrogen oxides content in torch combustion products, Journal of Physics:

Conference Series,. Vol. 944. No.1 (2018)

5. A. Mehrotra, S. Juttu, S. S. Ravishankar, R. P. Ghodke, J. Suryawanshi, Simultaneous Reduction of NOx and PM Emissions through Low Temperature EGR Cooling in Diesel Engines, s.1. : SAE Technical Paper 2014-01-2803, 50 (2014)

6. V. Jayaran, A. Nigan, W.A. Welch, J.W. Millar, I.I. Cocker, Effectiveness of emission control technologies for auxiliary engines on ocean -going vessels, Journal of the Air Waste Management Association, Vol. 61(1), pp. 14-21

7. By Nehemiah Sabinus Alozie and Lionel Christopher Ganippa. Diesel Exhaust Emissions and Mitigations, DOI: 10.5772/intechopen 85248 (2019)

8. A. R. Kulchitsky, Toxicity of automobile and tractor engines, M.: Academic project, 400 p (2004)

9. V. S. Kukis, V. A. Romanov, D. V. Shabalin, E. A. Omelchenko, The use of vortex tubes in a reciprocating internal combustion engine, SPb.: Izd-vo VA MTO, $215 \mathrm{p}$

10. A. I. Potapov, Ways to solve environmental problems of motor transport, $\mathrm{SPb} ., 568 \mathrm{p}$ (2006)

11. K. A. Morozov, Toxicity of automobile engines, M.: Legion-Avtodata, 80 p (2000) 